\title{
Metabolic Modeling of Pectobacterium parmentieri SCC3193 Provides Insights into Metabolic Pathways of Plant Pathogenic Bacteria
}

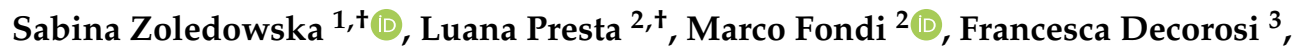 \\ Luciana Giovannetti ${ }^{3}$, Alessio Mengoni ${ }^{2, * \mathbb{C}}$ and Ewa Lojkowska ${ }^{1}$ \\ 1 Department of Biotechnology, Intercollegiate Faculty of Biotechnology, University of Gdansk and Medical \\ University of Gdansk, 58 Abrahama Street, 80-307 Gdansk, Poland; \\ sabina.zoledowska@biotech.ug.edu.pl (S.Z.); ewa.lojkowska@biotech.ug.edu.pl (E.L.) \\ 2 Department of Biology, University of Florence, via Madonna del Piano 6, Sesto Fiorentino, 50019 Florence, \\ Italy; luana.presta@unifi.it (L.P.); marco.fondi@unifi.it (M.F.) \\ 3 Department of Agri-food Production and Environmental Sciences, University of Florence, Piazzale delle \\ Cascine 18, 50144 Florence, Italy; francesca.decorosi@unifi.it (F.D.); luciana.giovannetti@unifi.it (L.G.) \\ * Correspondence: alessio.mengoni@unifi.it; Tel.: +055-457-4738 \\ + These authors contributed equally to this work.
}

Received: 17 February 2019; Accepted: 2 April 2019; Published: 5 April 2019

\begin{abstract}
Understanding plant-microbe interactions is crucial for improving plants' productivity and protection. Constraint-based metabolic modeling is one of the possible ways to investigate the bacterial adaptation to different ecological niches and may give insights into the metabolic versatility of plant pathogenic bacteria. We reconstructed a raw metabolic model of the emerging plant pathogenic bacterium Pectobacterium parmentieri SCC3193 with the use of KBase. The model was curated by using inParanoind and phenotypic data generated with the use of the OmniLog system. Metabolic modeling was performed through COBRApy Toolbox v. 0.10.1. The curated metabolic model of P. parmentieri SCC3193 is highly reliable, as in silico obtained results overlapped up to $91 \%$ with experimental data on carbon utilization phenotypes. By mean of flux balance analysis (FBA), we predicted the metabolic adaptation of P. parmentieri SCC3193 to two different ecological niches, relevant for the persistence and plant colonization by this bacterium: soil and the rhizosphere. We performed in silico gene deletions to predict the set of essential core genes for this bacterium to grow in such environments. We anticipate that our metabolic model will be a valuable element for defining a set of metabolic targets to control infection and spreading of this plant pathogen.
\end{abstract}

Keywords: Flux Balance Analysis; plant pathogenic bacteria; bacterial adaptation; metabolic reactions

\section{Introduction}

Plant-bacteria interplays have been studied for a long time, mainly regarding pathogenic and beneficial (symbiotic) interactions. Various details are now known concerning the molecular basis of all these interactions [1]. For example, biological studies of plant pathogenic bacteria allowed understanding the modulation of bacterial recognition by the plants and revealed important aspects of plant immune responses [2]. Furthermore, additional investigations have confirmed that plant pathogenic bacteria exploit high flexibility in utilization of different kinds of sugar, nitrogen, and phosphorus resources while adapting to the new environment; e.g., bacterial plant pathogen Pseudomonas syringae pv. tomato explicitly employs amino acid and sugar transporters to gain access to nutrients present in the environment [3]. Subsequently, during infection processes of the tomato plants, it utilizes resources within the host directly from the apoplast fluid [4]. 
To access the nutrients present in the plant tissues, pathogenic bacteria colonize, invade, and, later on, establish chronic infections within host plants. During the infection process, they enter plant tissues, through either wounds or natural openings, and occupy the apoplast of tissues or the xylem, where they multiply and spread [5]. Phytopathogenic microorganisms cause damage and often impair plant growth and reproduction. On the other hand, to defend themselves against microbiological invasion, plants rely on two kinds of innate immunity, i.e., via pathogen triggered immunity (PTI), which corresponds to pathogen perception via the recognition of conserved pathogen-associated molecular patterns and effector-triggered immunity (ETI) based on the recognition of pathogen effectors, molecules synthesized by the pathogens and delivered in the extracellular matrix or into the plant cell to enhance pathogen fitness [6].

To achieve compatible interaction, microorganisms at first need to overcome the plant's defenses that could abort the infection. Plant pathogenic bacteria, like Pectobacterium parmentieri, combine numerous strategies to accomplish that goal; e.g., they rely on the quorum sensing system [7].

P. parmentieri are pectinolytic bacteria belonging to Pectobacteriaceae family (known as Soft Rot Pectobacteriaceae, SRP) $[8,9]$, which are causative agents of soft rot in economically important crops such as potato, tomato, and maize [10]. Also, they are responsible for the blackleg disease, so far reported only on potato plants [11]. These Gram(-), rod-shaped bacteria are necrotrophs, able to destroy plant tissue components through the activity of Plant Cell Wall Degrading Enzymes (PCWDE) such as pectinases, cellulases, and proteases, secreted via Type I or II secretion system [12,13]. Interestingly, bacteria belonging to this species can produce different levels of the above-mentioned enzymes [14]. However, to cause disease symptoms those bacteria require favorable environmental conditions, such as humidity, nutrient availability accessed via natural plant openings, and preferably lower host resistance [11]. On the other hand, they can reside inside plant tissues as endophytes for a long time [11]. It is worth mentioning that bacteria from the genus Pectobacterium have been included among the ten most significant bacterial plant pathogens based on their economic impact [15] since crop losses caused by phytopathogenic microorganisms can reach up to $20 \%$ of total yield [16].

There is still limited knowledge regarding the cascade of genes expressed before and during the infection process in P. parmentieri. It was previously reported that initialization of infection progress is controlled by quorum sensing in closely related Pectobacterium atrosepticum [17]. Moreover, massive production of butanediol during plant infection by bacteria of the genera Dickeya and Pectobacterium was reported [18]. However, metabolic pathways necessary for promoting bacterial multiplication before and during plant infection connected with carbon and other compounds utilization in P. parmentieri have received little attention so far.

Given the complexity of bacteria-host relationships, they cannot be adequately investigated only using classical microbiological and molecular methods; instead, the coupled use of Phenotype Microarrays, computational, large-scale, and systematic frameworks are advisable. Constraint-based metabolic modeling is now a promising way to interpret puzzling, heterogeneous bacterial phenotypes [19], especially those related to bacteria-host interaction [20]. Constraint-based approaches, and in particular Flux Balance Analysis (FBA) [21], have been shown to infer realistic growth phenotypes and are claimed to provide a systems biology view on multi-omics data, possibly allowing prediction of physiological changes and evolution of bacterial populations [22,23]. Recently, genome-scale metabolic model (GEM) reconstruction and FBA have been used for deciphering the metabolic adaption of environmental microbes following ecological parameters variation [24], or comparison of activated metabolic reactions during ecological niche change [25], as well as for providing insights into the metabolic adaptation in human and bacterial plant pathogens $[3,26]$. To the best of our knowledge, only in a few cases, FBA has been applied in understanding the metabolic adaptation of specific plant bacterial pathogens. Studies performed on Ralstonia solanacearum showed that the quorum-sensing-dependent regulatory protein PhcA32 controls the trade-off between virulence factor production and bacterial proliferation [27]. Also, metabolic modeling of P. carotovorum subsp. carotovorum PC1 revealed 19 potential bactericide targets through a comprehensive in silico gene-deletion study [26]. 
The aim of this work was the reconstruction of a highly curated metabolic model of the plant pathogenic bacterium P. parmentieri SCC 3193 and the exploitation of this model to putatively identify the metabolic pathways relevant for P. parmentieri fitness in two different ecological niches, i.e., soil and rhizosphere. We show that niche changes may lead to a metabolic reconstitution of carbon-related pathways in P. parmentieri SCC3193 and we spot the core-set of essential genes in the two examined conditions. Moreover, we anticipate the model itself will represent a valuable element, which will pave the way to both the knowledge-base of the strain's biology and novel, applied technologies, such as genetic engineering and synthetic biology experiments.

\section{Materials and Methods}

\subsection{Metabolic Network Reconstruction and Model Refinement}

A draft metabolic model was built using the KBase Narrative Interface (www.kbase.com) which supports the reconstruction of metabolic models in microbes based on functional protein annotations. The closed genome of Pectobacterium sp. SCC3193 (NCBI Reference Sequence: NC_017845.1) [28] was used as an entry for such a process. The KBase automatic gap-filling algorithm was then run to improve the model. Automatic gap-filling compares the set of reactions in a given metabolic model to a database of all known reactions and finds a minimal set of reactions enabling it to grow in a predefined media, meaning that it forces a minimum flux of 0.1 through the biomass reaction. In our case, the medium growth chosen was Carbon-Glycerol, since its composition, (Supplementary Table S4 in Supplementary Information 2) is very similar to M9, one of the designed growth media of our experimental conditions.

This process allowed adding 44 new reactions and made two already existing reactions reversible (all the 46 reactions are listed in Supplementary Data 1). Afterwards, the model was downloaded and locally expanded by embedding reactions of orthologous genes present in the model of the closely related organism Escherichia coli K-12 MG1655 [29]; precisely, 93 genes and 383 reactions. The reason for this further model expansion is that the KBase gap-filling process may have discarded such reactions since they may not have been required for growth in Carbon-Glycerol. However, the strain may require fulfillment of these functions under different growth conditions.

Since the majority of $E$. coli reactions found belonged to a specific cellular district, the periplasm, we added this compartment in P. parmentieri model. The correctness of all the transporters added was verified during the last step of the model refinement, (i.e., the comparison of in silico outcomes to physiological abilities of the strain obtained through Phenotype Microarray (Omnilog) Experiment), where we observed that all the added gene allowed the model to grow on a different carbon source correctly.

Finally, during this test phase, the metabolic reconstruction was further enriched with exchange reactions (these reactions are the boundaries of the systems, representing the supply/removal of metabolites from the extra-organism space, so they do not need any biochemical or experimental evidence to be appended). At this stage, the model still presented six discrepancies that we decided to fix with manual gap-filling. Precisely, since PM showed evidence of strain growth on amylotriose, dihydroxyacetone, glucose-6-phosphate, D-arabinose, $N$-acetyl-D-galactosamine, stachyose, and sucrose, we added gap-filling transporters for such compounds, and we named them after the missing metabolite, even if the gene-protein relationship for that function was absent. These gap-fillings are fictitious reactions enabling the model to replicate a known physiological property of the strain when gene annotations for such a function are missing or when the metabolites enter the cell through one or more nonspecific transporter. The qualitative results of the comparison are reported in Supplementary Data 1 where, for each compound used as a carbon source, we show the growth predictions yielded by the model before and after the last round of refinements. 


\subsection{Biomass}

No comprehensive description of the macromolecular composition of the P. parmentieri biomass is available in the literature. However, such data are available for E. coli [29] which, like Parmentieri genus, belongs to the Enterobacteriaceae family. Therefore, the generic Gram-negative biomass reaction embedded in the model by KBase was substituted with the more accurate and similar one of E. coli model [29] and set as the model's objective function in all the experiments performed in this work. Despite being an approximation, that change gives a more realistic framework to perform simulations and it is allowed by strains of phylogenetic proximity and similar compound usage (as from Biolog outcomes comparison).

The complete biomass composition is given in Supplementary Information 2.

\subsection{Metabolic Modeling}

Metabolic modeling was performed using COBRApy Toolbox version 0.10 .1 [30] and the Gurobi 7.0.2 solver (www.gurobi.com) with libSBML library v5.10, in Python 2.7 framework (Department of Bioengineering, University of California, San Diego, CA, USA). The scripts for running all the in silico analysis performed in this work are available in Supplementary Information 1.

\subsection{Bacterial Strains and Culture Conditions}

The bacterial strain used in this study is P. parmentieri reference strain SCC3193, isolated from potato tuber in Finland [9,31]. For high-throughput phenotypic characterization, bacteria were grown on TSA medium at $28^{\circ} \mathrm{C}$ for $24 \mathrm{~h}$. For EnVision ${ }^{\mathrm{TM}}$ experiment bacteria were first grown in LB at $28^{\circ} \mathrm{C}$ for $24 \mathrm{~h}$ with constant agitation (120 RPM), later on in M9 for $24 \mathrm{~h}$ with constant agitation (130 RPM).

\subsection{Experimental High-Throughput Phenotypic Characterization on P. parmentieri SCC3193}

For high-throughput phenotypic characterization of P. parmentieri SCC3193 Biolog Plates for Carbon, Nitrogen, and Phosphorus-Sulfur utilization assay were used (PM1and PM2A, PM3, and PM4, respectively). Overnight bacterial culture was transferred from TSA medium to $5 \mathrm{~mL}$ of $0.85 \% \mathrm{NaCl}$, and bacterial suspension was adjusted to $\mathrm{OD}_{600}$ equaling 0.1 Later, $1 \mathrm{~mL}$ of bacterial suspension was transferred to $11 \mathrm{~mL}$ of Minimal Salts medium (M9-C: $0.6 \% \mathrm{Na}_{2} \mathrm{HPO}_{4}, 0.3 \% \mathrm{KH}_{2} \mathrm{PO}_{4}, 0.05 \% \mathrm{NaCl}$, $0.1 \% \mathrm{NH}_{4} \mathrm{Cl}, 0.005 \%$ Yeast Extract) supplemented with $120 \mu \mathrm{L}$ of Biolog A dye. To inoculate wells in PM plates $100 \mu \mathrm{L}$ of described bacterial suspension was used. The measurement was carried out in OmniLog $^{\mathrm{TM}}$ for $46 \mathrm{~h}$. Each reaction was tested in triplicate, and the experiment was performed twice. Mean results were analyzed with DuctApe [32]. All the results are reported in Supplementary Data 5.

Nutrients metabolic assay with the use of EnVision ${ }^{\mathrm{TM}}$ plate reader was performed to cross-check high-throughput phenotypic characterization. M9 media supplemented with $20 \%$ of selected carbon sources: $\alpha$-D-glucose, D-xylose, and D-mannitol, D-fructose, and citric acid were prepared. P. parmentieri SCC 3193 was grown in LB medium overnight at $28^{\circ} \mathrm{C}$ with constant agitation (120 RPM). Afterward, overnight bacterial cultures were centrifuged and washed twice in sterile Ringer Buffer and later $\mathrm{OD}_{600}$ of inoculum was adjusted to 0.1 . Fifty $\mu \mathrm{L}$ of inoculum was transferred to $450 \mu \mathrm{L}$ of M9 supplemented with different carbon sources to establish growth curves. Bacteria were cultured with agitation set at $120 \mathrm{RPM}$ in $28^{\circ} \mathrm{C}$ in 24 -well plate in EnVision ${ }^{\mathrm{TM}}$ plate reader. Optical density measurement at $600 \mathrm{~nm}$ was performed every $30 \mathrm{~min}$. The quantification of wet bacterial biomass was performed after bacterial growth in $300 \mathrm{~mL}$ of the media mentioned above after $48 \mathrm{~h}$. Bacterial cultures were centrifuged at $10{ }^{\circ} \mathrm{C}$ with 10,000 RPM for 10 min (Eppendorf Centrifuge 5920 R, Eppendorf Ltd., Warsaw, Poland) and frozen for $24 \mathrm{~h}$. Afterward, the wet bacterial biomass was weighed.

\subsection{OmniLog ${ }^{\mathrm{TM}}$ Data Processing and Analysis}

PM1 and PM2A obtained data analysis was performed with DuctApe [32]. Activity index (AV) values were calculated following subtraction of the value obtained for blank well from that of inoculated 
wells, whereas plots of the growth curves are of the unblanked data. Bacterial growth with each compound was considered positive if the AV value was $\geq 3$. Growth phenotypes were defined as negative if the $\mathrm{AV}$ value was $\leq 2$ and following a manual inspection of the unblanked curves. All the results are reported in Supplementary Data 5.

\subsection{In Silico Environmental Representations}

In silico representations of the nutritional composition of the soil, and rhizosphere were derived from a previously published paper [25]. The composition of each ere medium used is reported in Supplementary Information 2 (M9, soil, and rhizosphere). The composition of carbon-glycerol medium used through the KBase gap-filling application is reported.

\subsection{Flux Balance Analysis and Flux Variability Analysis}

Flux distribution predictions were assessed by performing FBA in M9, soil, and rhizosphere media. We performed loopless-FBA to avoid net fluxes around a closed cycle in a network at steady state, known as loops.

Moreover, since FBA only predicts one flux distribution among all possible solutions, we also performed loopless-FVA (Flux Variability Analysis). Since in some cases, the range of reaction flux was unbounded (going from -1000 to 1000) we decided to filter out those reactions whose FVA predicted range was larger than the predicted FBA flux the following criteria:

(1) If $\mathrm{f}_{\mathrm{FBA}}<0$

$$
\mathrm{f}_{\mathrm{FVA}}, \min \geq 1.2^{*} \mathrm{f}_{\mathrm{FBA}} \wedge \mathrm{f}_{\mathrm{FVA}}, \max \leq 0.8^{*} \mathrm{f}_{\mathrm{FBA}}
$$

(2) If $\mathrm{f}_{\mathrm{FBA}}>0$

$$
\mathrm{f}_{\mathrm{FVA}}, \min \geq 0.8^{*} \mathrm{f}_{\mathrm{FBA}} \wedge \mathrm{f}_{\mathrm{FVA}}, \max \leq 1.2^{*} \mathrm{f}_{\mathrm{FBA}}
$$

Loopless FBA and FVA predictions for each reaction can be retrieved in Supplementary Data 3 or by running Supplementary Information 1.

\subsection{Model's Predictive Value Estimation}

M9 growth medium was simulated in silico by constraining the lower bound of import reactions for each of the compounds present in the medium (as reported in Supplementary Information 2). When reproducing the PM experiment in silico, the model's performances were considered as true positives (TP) if growth was obtained both in silico and in vivo, true negatives (TN) in case of nongrowth both in silico and in vivo, false positives (FP) if growth was obtained in silico but not in vivo, and false negatives (FN) if vice versa.

The reliability of the obtained predictions was then estimated according to the following parameters:

(3) Sensitivity $=\mathrm{TP} /(\mathrm{TP}+\mathrm{FN})$

(4) Specificity $=\mathrm{TN} /(\mathrm{TN}+\mathrm{FP})$

(5) Precision $(\mathrm{PPV})=\mathrm{TP} /(\mathrm{TP}+\mathrm{FP})$

(6) Negative predicted value $(\mathrm{NPV})=\mathrm{TN} /(\mathrm{TN}+\mathrm{FN})$

(7) Accuracy $=(\mathrm{TP}+\mathrm{TN}) /(\mathrm{TP}+\mathrm{TN}+\mathrm{FP}+\mathrm{FN})$

(8) $F$-score $=2($ precision $\times$ sensitivity $) /($ precision + sensitivity $)$

\subsection{Single Gene Deletion Analysis}

Using genome-scale metabolic networks (GEMs), gene knockouts can be simulated to identify those genes whose removal is likely to impair the organism's growth. Specifically, it is possible to simulate mutants by deleting each gene included in the metabolic reconstruction and testing 
the predicted effects on the microbe's growth. Through this strategy, it is possible to estimate the contribution of each gene to the fitness of the strain by calculating the growth ratio (GRratio) between the growth rate of the mutant model $\left(\mu_{\mathrm{KO}}\right)$ and the one of the wild-type $\left(\mu_{\mathrm{WT}}\right)$ as:

$$
\text { (9) } \mathrm{GR}_{\text {ratio }}=\mu_{\mathrm{KO}} / \mu_{\mathrm{WT}}
$$

The knocked-out gene was considered essential if $\mathrm{GR}_{\text {ratio }}=1$ and nonessential if $<1$. Minimization of Metabolic Adjustment (MOMA [33]) algorithm was chosen to perform such analyses.

\subsection{COG Analyses}

The WebMGA web server [34] was used to provide functional Cluster of Orthologous Genes (COG) annotations ( $p$-value cutoff of 0.001) to each gene in the model. The COG annotation for each gene associated with variable reactions during the transition between two niches was extracted from the WebMGA server. Biases were determined after standardizing by the number of genes in each class of variable genes. Statistical significance was determined using Pearson's Chi-squared tests. The complete list of COG annotations is available as Supplementary Data 6.

\section{Results}

\subsection{Validation of a Genome-Scale Metabolic Model of P. parmentieri SCC3193}

FBA was employed to test if the reconstructed model could accurately predict the ability of P. parmentieri to produce biomass on utilized carbon sources. In particular, after verifying their presence in the metabolic reconstruction, we tested 91 compounds previously used in PM experiment. Details on the predictions of metabolites usage (before and after refinements) are given in Supplementary Data 1. Agreement between PM and in silico results is marked as TP (true positive) and TN (true negative), while discordance is marked as FP (false positive) and FN (false negative). The majority of the initial FN was solved by just adding an exchange reaction to the draft model.

At the end of that refinement round, the final P. parmentieri model was termed iLP1245, by the nomenclature standard [35]. It includes 1245 genes (covering $28 \%$ of the total number of coding sequences in the genome, 4449), 2182 reactions, and 2080 metabolites. Importantly, such coverage is generally accepted for metabolic models, since they only represent the fraction of metabolic genes. A description of the model is reported in Table 1 and the genetic features captured within it are presented as a plot of abundance per COG category in Figure 1.

Table 1. Properties of P. parmentieri SCC3193 genome and model.

\begin{tabular}{cc}
\hline P. parmentieri SCC3193 Genome & \\
\hline Total genome size & 4821 \\
Total protein-coding genes & 4449 \\
Pseudo Genes & 266 \\
iLP1245 characteristics & \\
Total genes of P. parmentieri SCC3193 & 1245 \\
Genes & 1224 \\
Pseudo Genes & 21 \\
Gap-filling genes & 7 \\
Total reactions & 2182 \\
Gene-associated reactions & 2120 \\
Exchange reactions & 195 \\
Transport reactions & 324 \\
Spontaneous reactions & 55 \\
Total metabolites & 2080 \\
\hline
\end{tabular}




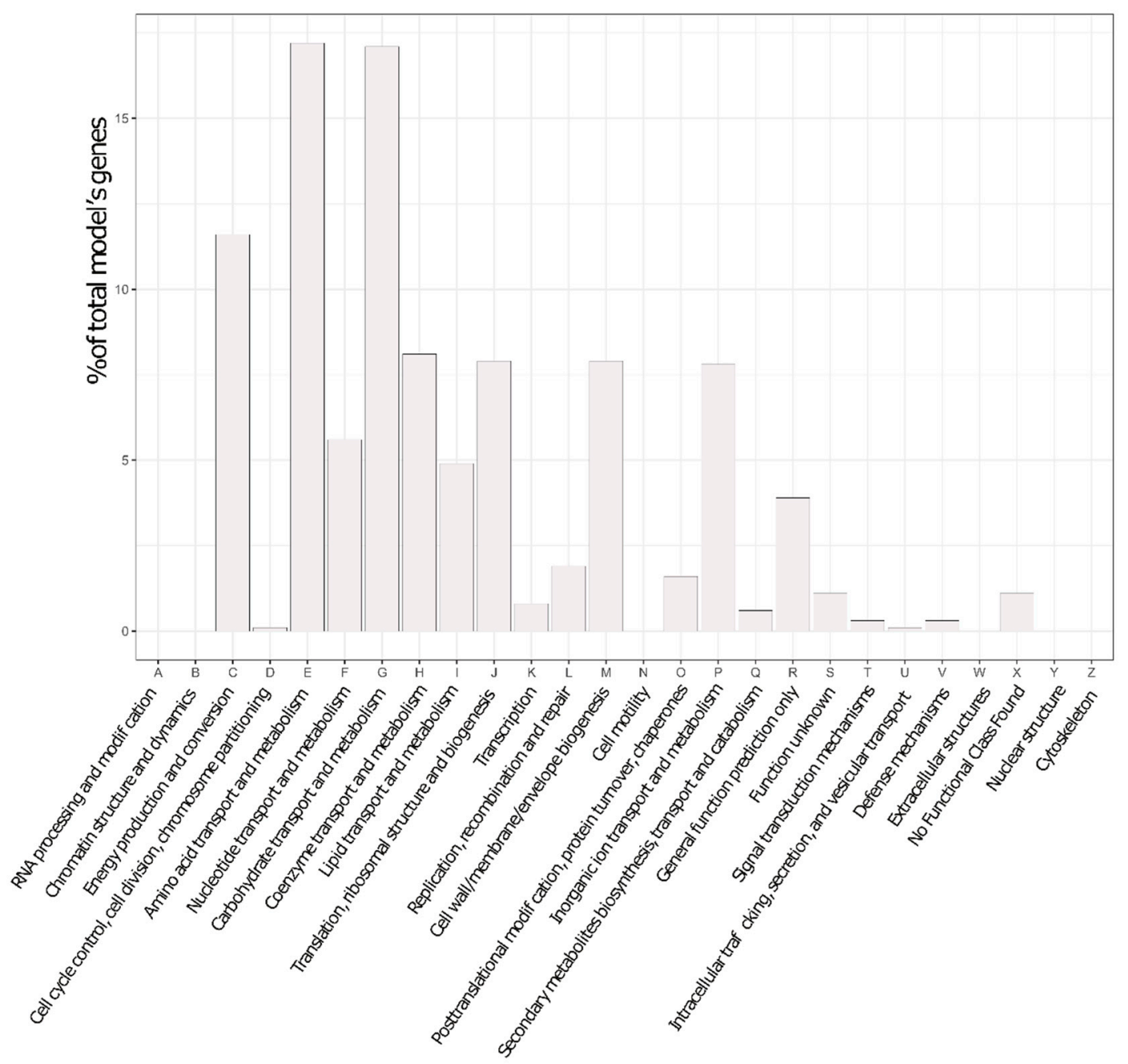

Figure 1. Genetic features captured by the model, according to Cluster of Orthologous Genes (COG) categories.

During in silico assessment, iLP1245 displayed agreement with PM in 83 out of 91 tested carbon substrates. As summarized in Figure 2, sensitivity, specificity, precision, accuracy, negative predictive value, and F-score (calculated as described previously [36], see materials and methods) reached very high scores, suggesting high reliability of the model.

Systems Biology, providing a unique, unambiguous, perennial, standard-compliant, and directly resolvable Markup Language (SBML) file of the model was validated by the online SBML validator tool (http://sbml.org/Facilities/Validator/), and is available as Supplementary Data 2. All the metabolites embedded in the model were annotated by using identifiers.org and the MIRIAM [37] registry to facilitate model reuse and search strategies, by identifiers. 


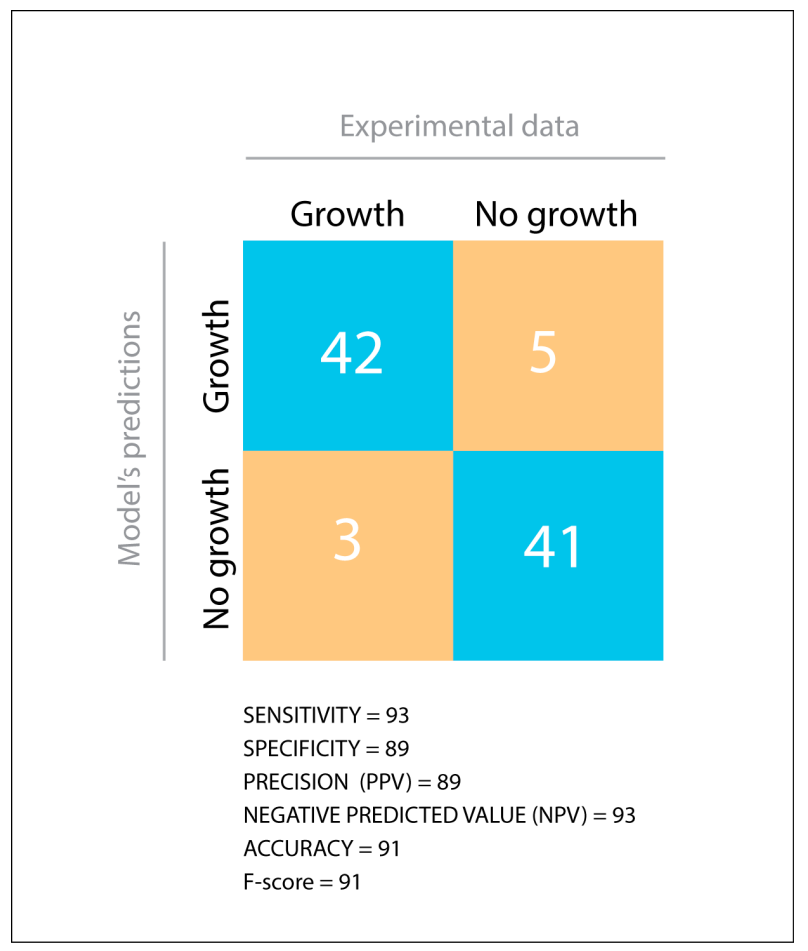

Figure 2. Comparison between Phenotype Microarray data and the model's predictions. TP $=$ True Positive, $\mathrm{TN}=$ True Negative, $\mathrm{FP}=$ False Positive, FN = False Negative. Statistical parameters were calculated as described in materials and methods.

\subsection{Phenotypic Characterization of P. parmentieri SCC3193 and Metabolic Model In-Depth Validation}

Phenotypical profiling with the use of Biolog Plates PM1, PM2A, PM3, and PM4 revealed that $P$. parmentieri SCC3193 could utilize all common sugar components of plant cell walls at high levels, e.g., sucrose, tartaric acid, D-cellobiose, stachyose, and, most importantly, pectin (Poly(1,4-alpha-D-galacturonide)) (Supplementary Data 1, Supplementary Data 5). The bacterium was very effectively exploiting D-Glucosamine and its derivatives ( $N$-Acetyl-D-Glucosamine, N-Acetyl-D-Galactosamine) together with xanthine (Supplementary Data 5). Khayi et al. 2016 [9] have proven that $P$. parmentieri strains can produce acid from raffinose and melibiose but not from malonic acid, and we also confirmed its ability to utilize raffinose and melibiose but not malonic acid by P. parmentieri in Biolog experiments (Supplementary Data 4). Besides, data about lactose, galactose, and maltose utilization were confirmed both in Biolog experiments and in silico simulations (Supplementary Data 1, Supplementary Data 4). Those results also agree with experimental data provided by Khayi et al. 2016 [9]. Moreover, metabolic abilities shown during PM experiments were compared to those obtained for other Enterobacteriaceae bacteria, including E. coli K12 MG1655, Pectobacterium carotovorum PC1 reported previously [26]. A comparison between these results is embedded in the Supplementary Data 1 file, and it highlights the metabolic similarity between the two Pectobacterium strains (80.53\% of agreement) and E. coli (71.06\% of agreement). Also, these findings support the biomass of P. parmentieri being approximated to that of E. coli (see materials and methods). We have also established of growth curves of P. parmentieri SCC3193 with randomly chosen carbon sources also analyzed in PM Microarray experiment, namely M9 medium supplemented with $\alpha$-D-glucose, D-fructose, and D-xylose. In general, the obtained results agree with the qualitative model's predictions (Supplementary Figure S1 and Supplementary Data 1).

\subsection{Differential Metabolic Adaptation to Soil and Rhizosphere Environment}

By analyzing flux changes in response to simulated environmental conditions (e.g., M9, soil, and the rhizosphere), it is possible to observe whether any significant metabolic rewiring occurs. Loopless 
FBA and loopless FVA (see Section 2) were used to estimate niche-specific metabolic adaptations in soil and the rhizosphere. The results obtained through FBA were cross-checked with FVA (see Supplementary Data 3 for results). Possible fluxes variations detected in the rhizosphere in respect to soil were interpreted as metabolic tuning occurring in the bacterium when it moves from one niche to the other. We focused only on the results for which variation is higher than $50 \%$ in rhizosphere compared to soil, as it was presented in a previous report [25].

The total amount of reactions taken into account was 208, corresponding to $\sim 10 \%$ of those embedded in the reconstruction. These were further classified in reactions with increased/decreased flux, and reactions turned on/off because of the environmental change (Figure 3). Notably, in this paper, we only present variations higher than $50 \%$; however, the number of reactions changing flux was estimated at several cutoffs, i.e., $10 \%, 20 \%, 30 \%, 40 \%$, and $50 \%$ of variation from the initial flux value (see Supplementary Figure S1 in Supplementary Information 2). Results indicate that the total number of reactions significantly changing the flux is slightly affected by the stringency of the cutoff, going from a maximum of 248 at $10 \%$ to a minimum of 208 at $50 \%$ cutoff, thus vouching for robustness of the analysis.

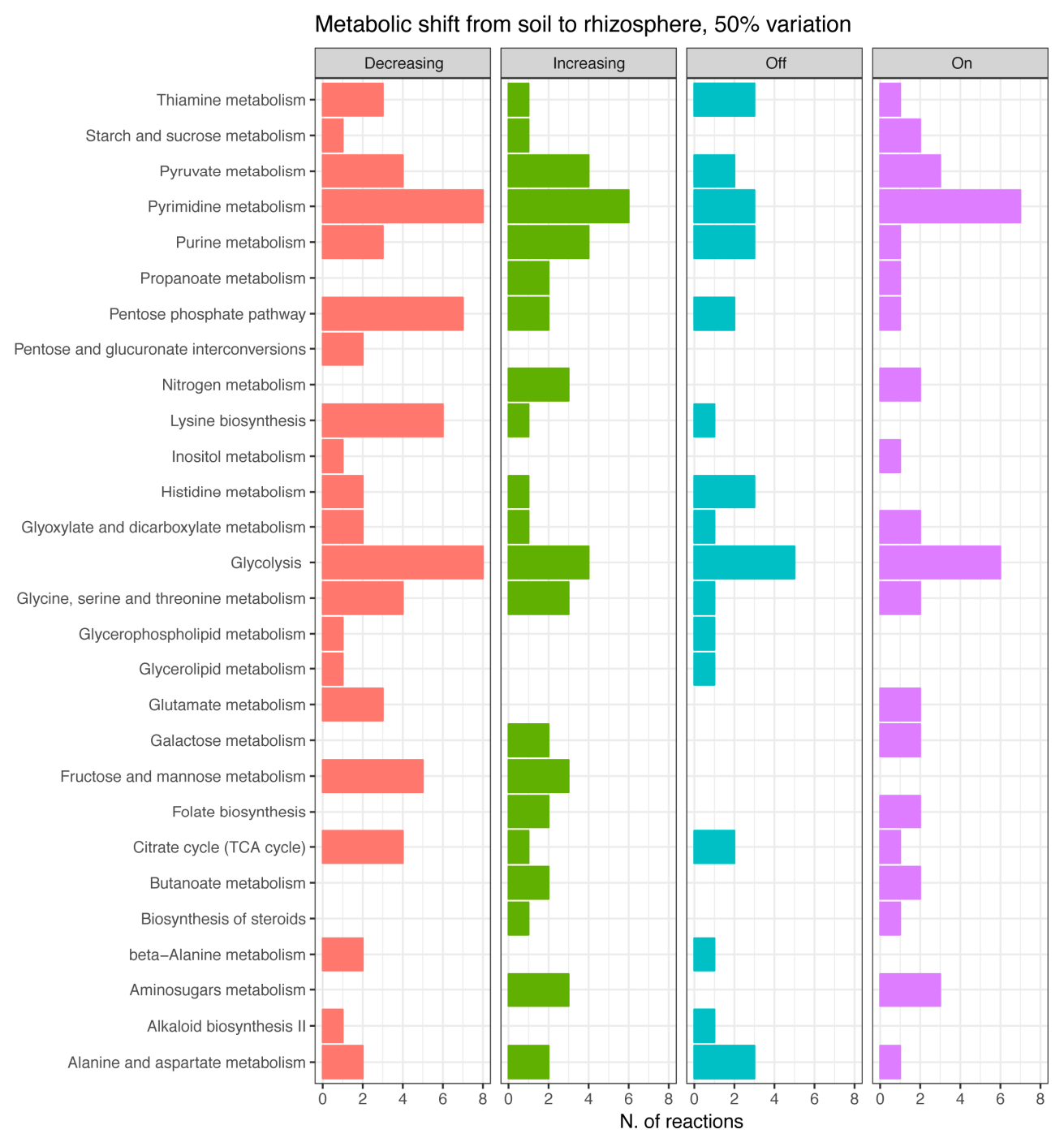

Figure 3. Reactions per metabolic pathways involved in the bacterial adaptation from soil to the rhizosphere. Flux changes higher than $50 \%$ are classified into four categories: decreasing, increasing, turning off, turning on. 
Based on that criterion, it appears that several pathways remain active in both examined conditions, though recruiting a different set of reactions. The primary distinction seems to occur in sugar metabolic pathways (Figure 3), tracking a metabolic adjustment from hexose and pentose phosphate to amino sugars' metabolism (amino sugars indeed are supposed to be more abundant in the rhizosphere [38]). Consequently, we can suggest that adaptation to the rhizosphere involves utilization of these carbon compounds [39] and that rhizosphere represents a rich environment for P. parmentieri, where these bacteria can thrive.

Also, some peculiar systems are turned on (Figure 3) in concomitance of such niche change, including nitrogen, butanoate, galactose, and propanoate metabolism, and biosynthetic pathways, including those of steroids and folate, indicating a specific adaptive response of P. parmentieri SCC3193 to the disparity of environmental (nutritional) setting. These compounds can either be precursors for volatiles compounds (VOCs) production or enter in VOC metabolic pathways; e.g., butanoate is an ester of butyric acid, which is among the most frequently secreted compounds $[18,40]$. VOCs could be strictly connected either with interbacterial communications or with virulence of plant pathogenic bacteria $[18,40]$. If so, this info could be used to develop pathogen biocontrol strategies.

Soil and Rhizosphere Adaptation, a Comparison with the Sinorhizobium meliloti Model

iLP1245 results in soil and rhizosphere were compared to those of the Sinorhizobium meliloti model [25], a plant symbiotic nitrogen-fixing bacterium, which, so far, is the only available GEM to be tested in such media. We observed that in P. parmentieri SCC 3193, only 10\% of reactions undergo a $50 \%$ flux change when comparing soil vs. rhizosphere; however, in S. meliloti, in the same conditions, more than the $20 \%$ of flux reactions was affected (including reactions which reversed flux direction). Moreover, in S. meliloti, $13 \%$ of active reactions were specific to just one of the environments, while only $5.3 \%$ of active reactions were environment-specific in P. parmentieri.

We can hypothesize that the smaller and more compact genome of $P$. parmentieri compared to the S. meliloti genome (4449 vs. 6204 protein-coding genes, respectively, including a multipartite genome organization in the latter species) allows a reduced metabolic redundancy for the first compared to the second [41] and a more generalist vs. specialist metabolic network (i.e., most reactions are not changing while the environment fluctuates). To test this hypothesis, we performed MOMA simulations of gene deletions (see Supplementary Data 4). The analyses revealed that P. parmentieri SCC3193 possesses an essential gene core composed of 241 metabolic genes, allowing growth in both soil and rhizosphere. Here, a set of eight genes was found to be essential in soil but not in the rhizosphere. This is in vast contrast to $S$. meliloti, where 66 genes were found as essential for growth in the same simulated rhizosphere environment [25], and supports the previously proposed hypothesis of a robust metabolic network of P. parmentieri SCC3193, which may allow the strain to rapidly accommodate relevant changes in environmental nutrient sources. Additionally, we can assume that the dense core of essential genes important for P. parmentieri allows bacteria of this species to quickly and effectively adapt to changing environmental conditions. This might explain why bacteria from this species can persist on plant residuals without interacting with host plant (potato) for extended periods, and subsequently, also the cosmopolitan lifestyle of this bacteria in the environment [10].

\subsection{In Silico Gene Deletions Provide Insight into the Fitness Relevance of Metabolic Modules}

Simulated single gene deletion is a very powerful in silico method for estimating a gene's knock-out fatality in as many settings as desired. Simulations on iLP1245 were made by using MOMA algorithm in three different media.

The number of essential genes found is 251 in M9 medium, 250 in soil, and 245 in the rhizosphere. The locus tag of such genes, alongside with their corresponding encoded protein, are reported in Supplementary Data 4. The Venn diagrams in Figure 4A show the overlap of EGs predicted among the three different conditions. Results indicate that a huge core of genes is likely to be mandatory in all the tested media, while only a small number stand out as essential in just one 
or two out of the three tested media. The method predicted the gene W5S_RS13875, encoding for DNA starvation/stationary phase protection protein (WP_014700482.1), as specifically required during growth in soil and rhizosphere, the gene W5S_RS15765, encoding for an ammonium transporter (WP_014700821.1), essential in M9 and rhizosphere, while eight genes appear to be specifically essential during growth in soil and M9 but not in rhizosphere. Seven of them belong to the thiamine and sulfur metabolism pathway: W5S_RS00965 (cystathionine gamma-synthase, WP_014698476.1), W5S_RS01140 (thiazole synthase ThiG, WP_012822036.1), W5S_RS01145 (sulfur carrier protein ThiS, WP_014698484.1), W5S_RS01150 (adenylyltransferase ThiF, WP_014698485.1), W5S_RS01155 (thiamine phosphate synthase ThiE, WP_014698486.1), W5S_RS01160 (phosphomethylpyrimidine synthase ThiC, WP_014698487.1), W5S_RS05940 (hydroxymethylpyrimidine/phosphomethylpyrimidine kinase ThiD, WP_014698998.1), while the last one, W5S_RS18250 (diaminopimelate decarboxylase, WP_043899153.1) is an enzyme involved in secondary metabolite production. Interestingly, the simulated rhizosphere growth medium contains thiamine which, conversely, is absent in soil and M9.

A

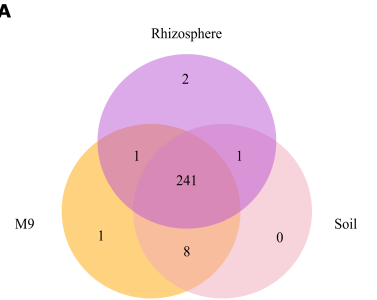

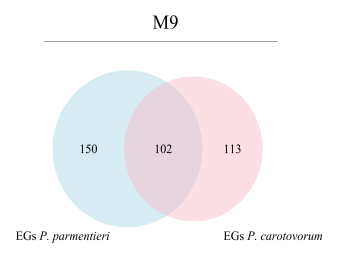

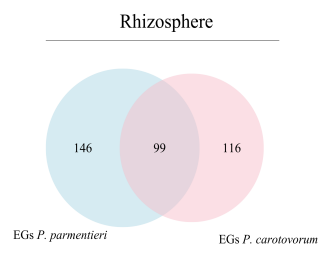

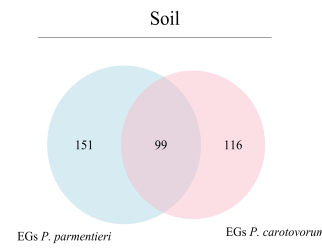

Figure 4. (A) Venn diagrams showing the amount of shared and unique essential genes in P. parmentieri SCC3193 for each of the examined conditions (M9, rhizosphere, and soil). (B) Venn diagrams showing the amount of shared and unique essential genes predicted by P. carotovorum PC1 model and P. parmentieri SCC3193 model for each of the examined conditions (M9, rhizosphere, and soil).

Three condition-specific genes have been predicted, one during growth in M9 medium, W5S_RS19605, encoding for class II fructose-bisphosphate aldolase (WP_014701539.1) and two during growth in the rhizosphere, W5S_RS19610 and W5S_RS06525, respectively encoding for a phosphoglycerate kinase (WP_005973111.1) and a long-chain fatty acid transporter (WP_014699104.1).

Moreover, we evaluated how the predicted EGs in iLP1245 compared to those predicted by the GEM reconstruction of Pectobacterium carotovorum PC1 [26], a closely related strain. Despite comparing EGs in different nutritional environments, we found a high level of overlap between the results given by the two models, as shown by Venn diagrams in Figure 4B. This supports the validity of EGs found through our simulation and, more in general, iLP1245 predictions consistency. As shown in Figure 4 and Supplementary Data 4, 99 genes are likely to be essential in P. parmentieri in all the simulated conditions, while seven are specifically essential in M9.

Importantly, we found out that nine genes, predicted as essentials by both models (listed in Table 2), were already marked as targets for new plants pathogens bactericides, since they share low sequence similarity both with their plant hosts and humans, ensuring safety requirement for agricultural field usage [26]. Moreover, these candidate targets are present in the therapeutic target database (TTD) [42], meaning that they are targets successfully used for human and animal pathogens, although they still have not been considered for crop disease treatments.

Since these nine genes are likely to be essential in both P. parmentieri SCC3193 and P. carotovorum subsp. carotovorum PC1, their essentiality should be tested in multiple Pectobacterium-like strains. 
Table 2. Candidate gene targets with no homology in humans and host and available in the therapeutic target database (TTD).

\begin{tabular}{cccc}
\hline Locus Gene & Candidate Drug Targets & Function & EC No. \\
\hline W5S_RS00975 & WP_012822018.1 & 5,10-methylenetetrahydrofolate reductase & 1.5 .1 .20 \\
W5S_RS01035 & WP_012822025.1 & UDP-N-acetylenolpyruvoylglucosamine reductase & 1.1 .1 .158 \\
W5S_RS01045 & WP_012822027.1 & pantothenate kinase & 2.7 .1 .33 \\
W5S_RS09185 & WP_014699609.1 & GTP cyclohydrolase I & 3.5 .4 .16 \\
W5S_RS10395 & WP_014699824.1 & NAD synthetase & 6.3 .1 .5 \\
W5S_RS13505 & WP_014700413.1 & Thymidylate kinase & 2.7 .4 .9 \\
W5S_RS06665 & WP_014699128.1 & bifunctional folylpolyglutamate synthase/ & dihydrofolate synthase \\
W5S_RS18995 & WP_014701427.1 & UDP-diphospho-muramoylpentapeptide & \\
W5S_RS19920 & WP_014701595.1 & beta-N-acetylglucosaminyltransferase & 2.4 .1 .227 \\
W5S_RS20855 & WP_005969274.1 & methionine synthase & 2.1 .2 .13 \\
W5S_RS22025 & WP_014701962.1 & aspartate-semialdehyde dehydrogenase & 1.2 .1 .11 \\
\hline
\end{tabular}

\section{Conclusions}

Plant-microbe interactions have been under intensive investigation in recent years, regardless of the nature of this communication: pathogenic or symbiotic. The ability to understand this interplay is connected with the complexity of the environment in which bacteria persist: soil, rhizosphere, or plant tissues. Metabolic modeling allows predicting and examining biochemical reactions involved in adaptation to the above-mentioned ecological niches as well as predicting the phenotypic outcomes of gene deletions. In this paper, for the first time, we report a high throughput experimental validation on the metabolic capability of P. parmentieri SCC3193 and a manually curated genome-scale metabolic model (GEM) of this plant pathogenic bacterium (iLP1245).

iLP1245 is highly reliable, obtaining $91 \%$ overlap in silico with large scale experimental data on carbon utilization phenotypes. This value perfectly fits with the currently accepted standard for GEMs [43,44]; for example, Pectobacterium carotovorum PC1 GEM showed an agreement of $80.4 \%$ with Phenotype Microarray (Biolog) experiments [26].

Moreover, we presented a broad set of EGs to be experimentally tested, nine of which are very likely to be used for bactericide design. This last result is significant since, at the moment, there is no available, efficient method for plant pathogenic bacteria eradication.

Supplementary Materials: The following are available online at http://www.mdpi.com/2076-2607/7/4/101/s1, Supplementary Figure S1. Growth curves and rates of P. parmentieri SCC3193. A: Growth curves of P. parmentieri SCC3193 in M9 media supplemented with different carbon sources over time invitro. B: Growth rates of P. parmentieri SCC 3193 obtained in vitro compared to in silico calculated growth rates basing on the metabolic model. Supplementary Information 1. The script is enabling to perform all the analysis in the manuscript. File type: python script. Supplementary Information 2. Supplementary Text, Supplementary Tables, and Supplementary Figures. File type: PDF document. Supplementary Data 1. Prediction on the usage of metabolites before and after the gap filling process. List of all reactions added/changed in the model during curation phases. Comparison of PM results between Escherichia coli K-12 MG1655, Pectobacterium carotovorum PC1, and Pectobacterium parmentieri SC3193. File type: Excel document. Supplementary Data 2. The SBML file of the model. File type: XML formatted file. Supplementary Data 3. Reactions Flux during growth in soil versus the rhizosphere according to loopless FBA and loopless FVA; File type: Excel document. Supplementary Data 4. All essential genes found with their encoded protein and function. Growth ratio calculated with FBA and MOMA is shown. EGs predicted by both Pectobacterium carotovorum PC1 model and Pectobacterium parmentieri SC3193 model. File type: Excel document. Supplementary Data 5. All Phenotype Microarray data generated in this study and dataset and plots generated through DuctApe program. File type: Excel document. Supplementary Data 6. The COG annotations for all genes included in the model, as generated by WebMGA. File type: Excel document.

Author Contributions: Conceptualization, A.M. and E.L.; Data curation, L.P.; Formal analysis, L.P.; Funding acquisition, S.Z. and E.L.; Investigation, S.Z., L.P. and F.D.; Validation, S.Z., L.P., M.F., L.G., A.M. and E.L.; Writing—original draft, S.Z. and L.P.; Writing—review \& editing, S.Z., L.P., A.M. and E.L.

Funding: This work was supported by the National Science Centre in Poland via grant no. 2014/14/M/NZ8/00501 awarded to E.L. and by the University of Gdansk in Poland via grant no. 538-M031-B187-16 awarded to S.Z. 
Conflicts of Interest: The authors declare no conflict of interest. The funders had no role in the design of the study; in the collection, analyses, or interpretation of data; in the writing of the manuscript, or in the decision to publish the results.

\section{References}

1. Bais, H.P.; Weir, T.L.; Perry, L.G.; Gilroy, S.; Vivanco, J.M. The role of root exudates in rhizosphere interactions with plants and other organisms. Annu. Rev. Plant Biol. 2006, 57, 233-266. [CrossRef] [PubMed]

2. Cui, H.; Xiang, T.; Zhou, J.-M. Plant immunity: A lesson from pathogenic bacterial effector proteins. Cell. Microbiol. 2009, 11, 1453-1461. [CrossRef]

3. Mithani, A.; Hein, J.; Preston, G.M. Comparative analysis of metabolic networks provides insight into the evolution of plant pathogenic and nonpathogenic lifestyles in Pseudomonas. Mol. Biol. Evol. 2011, 28, 483-499. [CrossRef] [PubMed]

4. Rico, A.; Preston, G.M. Pseudomonas syringae pv. tomato DC3000 uses constitutive and apoplast-induced nutrient assimilation pathways to catabolize nutrients that are abundant in the tomato apoplast. Mol. Plant Microbe Interact. 2008, 21, 269-282. [CrossRef] [PubMed]

5. Motyka, A.; Zoledowska, S.; Sledz, W.; Lojkowska, E. Molecular methods as tools to control plant diseases caused by Dickeya and Pectobacterium spp: A minireview. New Biotechnol. 2017, 39, 181-189. [CrossRef] [PubMed]

6. Chisholm, S.T.; Coaker, G.; Day, B.; Staskawicz, B.J. Host-Microbe Interactions: Shaping the Evolution of the Plant Immune Response. Cell 2006, 124, 803-814. [CrossRef] [PubMed]

7. Põllumaa, L.; Alamäe, T.; Mäe, A. Quorum sensing and expression of virulence in Pectobacteria. Sensors 2012, 12,3327-3349. [CrossRef]

8. Adeolu, M.; Seema, A.; Naushad, S.; Gupta, R.S. Genome-based phylogeny and taxonomy of the 'Enterobacteriales': Proposal for Enterobacterales ord. nov. divided into the families Enterobacteriaceae, Erwiniaceae fam. nov., Pectobacteriaceae fam. nov., Yersiniaceae fam. nov., Hafniaceae fam. nov., Morgane. Int. J. Syst. Evol. Microbiol. 2016, 66, 5575-5599.

9. Khayi, S.; Cigna, J.; Chong, T.M.; Quêtu-Laurent, A.; Chan, K.-G.; Hélias, V.; Faure, D. Transfer of the potato plant isolates of Pectobacterium wasabiae to Pectobacterium parmentieri sp. nov. Int. J. Syst. Evol. Microbiol. 2016, 66, 5379-5383.

10. Toth, I.K.; Bell, K.S.; Holeva, M.C.; Birch, P.R.J. Soft rot Erwiniae: From genes to genomes. Mol. Plant Pathol. 2003, 4, 17-30. [CrossRef]

11. Pérombelon, M.C.M. Potato diseases caused by soft rot erwinias: An overview of pathogenesis. Plant Pathol. 2002, 51, 1-12. [CrossRef]

12. Ma, B.; Hibbing, M.E.; Kim, H.-S.; Reedy, R.M.; Yedidia, I.; Breuer, J.J.J.; Breuer, J.J.J.; Glasner, J.D.; Perna, N.T.; Kelman, A.; et al. Host range and molecular phylogenies of the soft rot enterobacterial genera Pectobacterium and Dickeya. Phytopathology 2007, 97, 1150-1163. [CrossRef] [PubMed]

13. Charkowski, A.; Blanco, C.; Condemine, G.; Expert, D.; Franza, T.; Hayes, C.; Hugouvieux-Cotte-Pattat, N.; Solanilla, E.L.; Low, D.; Moleleki, L.; et al. The Role of Secretion Systems and Small Molecules in Soft-Rot Enterobacteriaceae Pathogenicity. Annu. Rev. Phytopathol. 2012, 50, 425-449. [CrossRef]

14. Zoledowska, S.; Motyka, A.; Zukowska, D.; Sledz, W.; Lojkowska, E. Population Structure and Biodiversity of Pectobacterium parmentieri Isolated from Potato Fields in Temperate Climate. Plant Dis. 2018, 102, $154-164$. [CrossRef] [PubMed]

15. Mansflieds, J.; Genin, S.; Magor, S.; Citovsky, V.; Sriariyanum, M.; Ronald, P.; Dow, M.; Verdier, V.; Beer, S.V.; Machado, M.A.; et al. Top 10 plant pathogenic bacteria in molecular plant pathology. Mol. Plant Pathol. 2012, 13, 614-629. [CrossRef] [PubMed]

16. Czajkowski, R.; Pérombelon, M.C.M.; van Veen, J.A.; van der Wolf, J.M. Control of blackleg and tuber soft rot of potato caused by Pectobacterium and Dickeya species: A review. Plant Pathol. 2011, 60,999-1013. [CrossRef]

17. Liu, H.; Coulthurst, S.J.; Pritchard, L.; Hedley, P.E.; Ravensdale, M.; Humphris, S.; Burr, T.; Takle, G.; Brurberg, M.-B.; Birch, P.R.J.; et al. Quorum sensing coordinates brute force and stealth modes of infection in the plant pathogen Pectobacterium atrosepticum. PLoS Pathog. 2008, 4, e1000093. [CrossRef] 
18. Effantin, G.G.; Rivasseau, C.; Gromova, M.; Bligny, R.; Hugouvieux-Cotte-Pattat, N. Massive production of butanediol during plant infection by phytopathogenic bacteria of the genera Dickeya and Pectobacterium. Mol. Microbiol. 2011, 82, 988-997. [CrossRef]

19. Oberhardt, M.A.; Palsson, B.Ø.; Papin, J.A. Applications of genome-scale metabolic reconstructions. Mol. Syst. Biol. 2009, 5, 320. [CrossRef]

20. Duan, G.; Christian, N.; Schwachtje, J.; Walther, D.; Ebenhöh, O. The Metabolic Interplay between Plants and Phytopathogens. Metabolites 2013, 3, 1-23. [CrossRef]

21. Orth, J.D.; Thiele, I.; Palsson, B.Ø. What is flux balance analysis? Nat. Biotechnol. 2010, 28, 245-248. [CrossRef] [PubMed]

22. Großkopf, T.; Consuegra, J.; Gaffé, J.; Willison, J.C.; Lenski, R.E.; Soyer, O.S.; Schneider, D. Metabolic modelling in a dynamic evolutionary framework predicts adaptive diversification of bacteria in a long-term evolution experiment. BMC Evol. Biol. 2016, 16, 163. [CrossRef] [PubMed]

23. Angione, C.; Lió, P.; Dobzhansky, T.; Romero, I.G.; Ruvinsky, I.; Gilad, Y.; Fraser, H.B.; Pál, C.; Papp, B.; Lercher, M.J.; et al. Predictive analytics of environmental adaptability in multi-omic network models. Sci. Rep. 2015, 5, 15147. [CrossRef] [PubMed]

24. Fondi, M.; Bosi, E.; Giudice, A.L.; Fani, R. A Systems Biology View on Bacterial Response to Temperature Shift. In Biotechnology of Extremophiles; Springer: Cham, Switzerland, 2016; pp. 597-618, ISBN 978-3-319-13520-5.

25. diCenzo, G.C.; Checcucci, A.; Bazzicalupo, M.; Mengoni, A.; Viti, C.; Dziewit, L.; Finan, T.M.; Galardini, M.; Fondi, M.; Harrison, P.W.; et al. Metabolic modelling reveals the specialization of secondary replicons for niche adaptation in Sinorhizobium meliloti. Nat. Commun. 2016, 7, 12219. [CrossRef]

26. Wang, C.; Deng, Z.-L.; Xie, Z.-M.; Chu, X.-Y.; Chang, J.-W.; Kong, D.-X.; Li, B.-J.; Zhang, H.-Y.; Chen, L.-L. Construction of a genome-scale metabolic network of the plant pathogen Pectobacterium carotovorum provides new strategies for bactericide discovery. FEBS Lett. 2015, 589, 285-294. [CrossRef] [PubMed]

27. Peyraud, R.; Cottret, L.; Marmiesse, L.; Gouzy, J.; Genin, S.; Brown, S.; Palmer, K.; Whiteley, M.; Eisenreich, W.; Dandekar, T.; et al. A Resource Allocation Trade-Off between Virulence and Proliferation Drives Metabolic Versatility in the Plant Pathogen Ralstonia solanacearum. PLoS Pathog. 2016, 12, e1005939. [CrossRef] [PubMed]

28. Koskinen, J.P.; Laine, P.; Niemi, O.; Nykyri, J.; Harjunpää, H.; Auvinen, P.; Paulin, L.; Pirhonen, M.; Palva, T.; Holm, L.; et al. Genome sequence of Pectobacterium sp. strain SCC3193. J. Bacteriol. 2012, 194, 6004. [CrossRef]

29. Feist, A.M.; Henry, C.S.; Reed, J.L.; Krummenacker, M.; Joyce, A.R.; Karp, P.D.; Broadbelt, L.J.; Hatzimanikatis, V.; Palsson, B.Ø. A genome-scale metabolic reconstruction for Escherichia coli K-12 MG1655 that accounts for 1260 ORFs and thermodynamic information. Mol. Syst. Biol. 2007, 3, 121. [CrossRef]

30. Schellenberger, J.; Que, R.; Fleming, R.M.T.; Thiele, I.; Orth, J.D.; Feist, A.M.; Zielinski, D.C.; Bordbar, A.; Lewis, N.E.; Rahmanian, S.; et al. Quantitative prediction of cellular metabolism with constraint-based models: The COBRA Toolbox v2.0. Nat. Protoc. 2011, 6, 1290-1307. [CrossRef]

31. Nykyri, J.; Niemi, O.; Koskinen, P.; Nokso-Koivisto, J.; Pasanen, M.; Broberg, M.; Plyusnin, I.; Törönen, P.; Holm, L.; Pirhonen, M.; et al. Revised phylogeny and novel horizontally acquired virulence determinants of the model soft rot phytopathogen Pectobacterium wasabiae SCC3193. PLoS Pathog. 2012, 8, e1003013. [CrossRef]

32. Galardini, M.; Mengoni, A.; Biondi, E.G.; Semeraro, R.; Florio, A.; Bazzicalupo, M.; Benedetti, A.; Mocali, S. DuctApe: A suite for the analysis and correlation of genomic and OmniLog ${ }^{\text {TM }}$ Phenotype Microarray data. Genomics 2014, 103, 1-10. [CrossRef] [PubMed]

33. Segre, D.; Vitkup, D.; Church, G.M.; Segrè, D.; Vitkup, D.; Church, G.M. Analysis of optimality in natural and perturbed metabolic networks. Proc. Natl. Acad. Sci. USA 2002, 99, 15112-15117. [CrossRef] [PubMed]

34. Wu, S.; Zhu, Z.; Fu, L.; Niu, B.; Li, W. WebMGA: A customizable web server for fast metagenomic sequence analysis. BMC Genom. 2011, 12, 444. [CrossRef]

35. Reed, J.L.; Vo, T.D.; Schilling, C.H.; Palsson, B.O. An expanded genome-scale model of Escherichia coli K-12 (iJR904 GSM/GPR). Genome Biol. 2003, 4, R54. [CrossRef] [PubMed]

36. Mendoza, S.N.; Cañón, P.M.; Contreras, Á.; Ribbeck, M.; Agosín, E. Genome-Scale Reconstruction of the Metabolic Network in Oenococcus oeni to Assess Wine Malolactic Fermentation. Front. Microbiol. 2017, 8, 534. [CrossRef] [PubMed]

37. Juty, N.; Le Novere, N.; Laibe, C. Identifiers.org and MIRIAM Registry: Community resources to provide persistent identification. Nucleic Acids Res. 2012, 40, D580-D586. [CrossRef] [PubMed] 
38. Sørensen, J.; Jensen, L.E.; Nybroe, O. Soil and rhizosphere as habitats for Pseudomonas inoculants: New knowledge on distribution, activity and physiological state derived from micro-scale and single-cell studies. Plant Soil 2001, 232, 97-108. [CrossRef]

39. Gorshkov, V.; Kwenda, S.; Petrova, O.; Osipova, E.; Gogolev, Y.; Moleleki, L.N. Global gene expression analysis of cross-protected phenotype of Pectobacterium atrosepticum. PLoS ONE 2017, 12, e0169536. [CrossRef]

40. Kanchiswamy, C.N.; Malnoy, M.; Maffei, M.E. Chemical diversity of microbial volatiles and their potential for plant growth and productivity. Front. Plant Sci. 2015, 6, 151. [CrossRef]

41. diCenzo, G.C.; Finan, T.M. Genetic redundancy is prevalent within the $6.7 \mathrm{Mb}$ Sinorhizobium meliloti genome. Mol. Genet. Genom. 2015, 290, 1345-1356. [CrossRef]

42. Li, Y.H.; Yu, C.Y.; Li, X.X.; Zhang, P.; Tang, J.; Yang, Q.; Fu, T.; Zhang, X.; Cui, X.; Tu, G.; et al. Therapeutic target database update 2018: Enriched resource for facilitating bench-to-clinic research of targeted therapeutics. Nucleic Acids Res. 2018, 46, D1121-D1127. [PubMed]

43. Thiele, I.; Palsson, B.Ø. A protocol for generating a high-quality genome-scale metabolic reconstruction. Nat. Protoc. 2010, 5, 93-121. [CrossRef] [PubMed]

44. Chan, S.H.J.; Cai, J.; Wang, L.; Simons-Senftle, M.N.; Maranas, C.D. Standardizing biomass reactions and ensuring complete mass balance in genome scale metabolic models. Bioinformatics 2017, 33, 3603-3609. [CrossRef] [PubMed]

(C) 2019 by the authors. Licensee MDPI, Basel, Switzerland. This article is an open access article distributed under the terms and conditions of the Creative Commons Attribution (CC BY) license (http://creativecommons.org/licenses/by/4.0/). 